\title{
Relief of hot flashes with escitalopram in non-depressed menopausal women in Japan: Results of a retrospective analysis
}

\author{
Chikako Mori ${ }^{1}$, Atsushi Imai ${ }^{2 *}$ \\ ${ }^{1}$ Clerical Support Unit for Physicians, Matsunami General Hospital, Gifu, Japan \\ ${ }^{2}$ Institute of Endocrine-Related Cancer, Matsunami General Hospital, Gifu, Japan; \\ *Corresponding Author: aimai@matsunami-hsp.or.jp
}

Received 4 September 2012; revised 3 October 2012; accepted 12 October 2012

\begin{abstract}
Purpose: Hormone therapy (estrogen with or without progestin) remains the gold standard treatment for hot flashes in menopausal women, but concerns for the risk of hormone therapy have resulted in its decline and a demand for nonhormonal treatments with demonstrated efficacy for hot flashes. Aim of this study was to examine the efficacy of selective serotonin reuptake inhibitor escitalopram on hot flashes in a healthy sample of non-depressant menopausal women in Japan. Methods: We retrospectively analyzed the medical records of $\mathbf{1 1}$ menopausal patients with hot flashes, who received escitalopram (10 mg daily) for 2 weeks between March and August 2012. Hot flashes severities and scores were recorded on a scale of 0 to 10 points, at beginning and end of 2 weeks treatment. Results: At 2 weeks of therapy, 9 of 11 patients reported significant decreases in hot flash frequency and severity, but the remission of the symptom was not observed in 2 patients. Speed of relief from hot flashes was rapid (within one week). Conclusions: Escitalopram $10 \mathrm{mg} /$ day may be a prompt and effective option for treating hot flashes in menopausal women who do not want to use hormone replacement therapy.
\end{abstract}

Keywords: Hot Flashes; Escitalopram; Selective Serotonin Reuptake Inhibitors; Menopause

\section{INTRODUCTION}

Hot flashes are the predominant menopausal symptoms, with up to $80 \%-90 \%$ of women reporting hot flashes around menopause [1,2]. Because many of the physiological changes that occur during menopause result from decreased levels of estrogen (estradiol level $<10 \mathrm{pg} / \mathrm{ml}$ ), hormone replacement therapy (estrogen with or without progestin) has historically been considered as a first-line treatment for hot flashes. However, hormonal treatments, despite the fact that they represent the most effective therapies, are not used for the treatment of hot flashes in theses women because of concerns that they may increase for estrogen-dependent cancers (e.g. breast cancer and endometrial cancer) [3,4]. A number of recent studies have raised concerns about the other adverse effects of hormone replacement therapy; increased cardiovascular and thromboembolic risk [5], increased risk of ovarian cancer [6] and dementia [7], and the numerous side effects such as atypical bleeding and edema [4,8-10].

Therefore many women who have concerns regarding the safety of hormonal therapy are seeking novel nonhormonal interventions to help them cope with menopausal hot flashes. The nonhormonal prescription therapy has been studied mostly in women with breast cancer and endometrial cancer or who are at risk for these cancers for their effectiveness at relieving hot flashes. Some previous literatures have examined the use of a anti-depressant (selective serotonin reuptake inhibitor (SSRI) [11-14] and a g-aminobutyric acid analog $[12,15]$ to treat hot flashes in a psychologically healthy sample of women (see for Refs. [11-14]). We conducted to retrospectively analyze the efficacy of escitalopram, the newest SSRI, in alleviating the frequency and severity of hot flashes. Escitalopram was selected because it is well-tolerated and has little drug-drug interaction [11] but has not been systematically studied as a treatment of hot flashes in Japan.

\section{METHODS}

Between March and August 2012, 81 new patients with bothersome hot flashes, defined by occurrence at least 20 times per week and sufficient severity to make the patient desire therapeutic intervention were entered onto our database. Patients with current or planned use of any agents for hot flashes (e.g. estrogens, androgens, gaba- 
pentin, pregabalin, antidepressants) were excluded for the analysis. Patients were asked to complete questionnaires by themselves or with assistance. In addition to the hot flash diaries, patients were asked to complete a symptom experience questionnaire at baseline and the end of week 2. This questionnaire asked patients to score, on a scale of 0 to 10 points, the following symptoms; sleepiness, nausea, dizziness, fatigue, abnormal sweating, constipation, mouth dryness, blurred or double vision, less than desirable libido.

In this retrospective chart review, 11 patients who do not want to use hormone replacement therapy initiated treatment with escitalopram $10 \mathrm{mg} /$ day for $2-4$ weeks at the age of 45 - 58 years. If patients developed symptoms that were of unacceptable severity at 2 weeks, escitalopram stopped or changed to estradiol. All subjects provided written informed consents against these therapy protocols.

Paired t-tests were used to analyze in each hot flashes score change from baseline. Statistical significance was defined as $\mathrm{P}<0.05$.

\section{RESULTS}

Hot flash score and frequency changes during treatment week 2 were available for all 11 patients. Baseline characteristics of subjects are outlined in Table 1. At baseline, the mean score of hot flashes was 9.78, and escitalopram $10 \mathrm{mg} /$ day significantly reduced the frequency. Hot flash score decreases from baseline, assessed by a scale of 0 to 10 points, are also listed in Table 1 . Nine of 11 women $(81.8 \%)$ were treatment responders (greater than $50 \%$ decrease in hot flashes score). Speed of relief from hot flashes was rapid (within one week). Improvements in hot flashes were observed after 2 week of active treatment and were maintained throughout the 8 weeks treatment period (data not shown). The remission of the symptom was not observed in 2 patients (cases 10 and 11).

\section{DISCUSSION}

This retrospective analysis suggests that escitalopram is an effective and rapid non-hormonal approach for the treatment of hot flashes and maybe other symptoms associated with menopause in Japanese women, who tend to concern the health risk associated with hormone replacement therapy.

Four pilot or randomized placebo-controlled studies have compared with placebo for treatment of hot flashes [4,16-18]. First study enrolled 25 menopausal women, with no significant psychiatric or medical history. All women were treated with escitalopram $(10-20 \mathrm{mg}$ flexibly dosed) for 8 weeks. The active treatment was preceded by a single blind placebo lead-in period. Sixteen women $(64 \%)$ were treatment responders (grater than $50 \%$ decrease in hot flash frequency) with an average decrease of $55 \%$ in hot flash frequency and severity and improvements in dysphoria, anxiety, quality of life, and sleep [4]. In a multicenter, double-blind, placebocontrolled, parallel group RCT by Freeman et al. $(\mathrm{n}=194 ; 8$ weeks) [16], 55\% of the escitalopram group (versus $36 \%$ of the placebo group) reported $\geq 50 \%$ decreased in both hot flash frequency and severity. Overall discontinuation due to side effects (e.g. dizziness/lightheaded, vivid dreams, nausea, excessive sweating) was $4 \%$. Notably, women who were not depressed or anxious responded swiftly to escitalopram, with significantly greater improvement compared to placebo after one week of treatment. Soares et al. [17] examined the efficacy of escitalopram versus estrogen and progestogen therapy for the treatment symptomatic periand postmenopausal women ( $\mathrm{n}=40,8$ weeks) Escitaropram (flexible dose, 10 -

Table 1. Baseline characteristics of subjects and changes in hot flashes scores during escitalopram treatment.

\begin{tabular}{cccccc}
\hline \multirow{2}{*}{ Case no. } & Age (years) & Weight $(\mathrm{kg})$ & \multicolumn{2}{c}{ Hot flashes scores } & \multirow{2}{*}{ Week 2/Baseline } \\
\cline { 4 - 5 } 1 & 61 & 57 & Baseline & Week 2 & 0.5 \\
2 & 63 & 64 & 9 & 3 & 0.33 \\
3 & 53 & 70 & 10 & 3 & 0.3 \\
4 & 49 & 69 & 7 & 3 & 0.43 \\
5 & 49 & 53 & 10 & 5 & 0.5 \\
6 & 54 & 50 & 10 & 3 & 0.33 \\
7 & 52 & 45 & 9 & 2 & 0.22 \\
8 & 52 & 52 & 10 & 4 & 0.4 \\
9 & 54 & 71 & 10 & 3 & 0.3 \\
10 & 58 & 68 & 9 & 9 & 8 \\
Mean \pm SD & $53.9 \pm 4.9$ & $59.5 \pm 9.2$ & $9.2 \pm 0.98$ & $4.3 \pm 2.24^{*}$ & 0.89 \\
\hline
\end{tabular}

${ }^{*} \mathrm{P}<0.01$ versus baseline. 
$20 \mathrm{mg} /$ day; fixed dose $10 \mathrm{mg}$ /day for the first 4 weeks) is more efficacious that estrogen and progestogen (ethinyl estradiol $5 \mu \mathrm{g}$ /day plus norethindrone acetate $1 \mathrm{mg}$ /day). Their another randomizised, double-blind study showed comparable efficacy of escitalopram to desvenlafaxine [18]. In theses studies, escitalopram has not been mentioned in bone mineral density decline.

Lastly, we reported successful management of a series of Japanese patients with hot flashes by use of a SSRI escitalopram, although there are three important limitations to the current analysis that warrant consideration. The small sample size may limit the generalizability of study findings. In addition, as this retrospective study was uncontrolled, the role of chance as well as non-specific treatment factors could not be explored. High placebo responses rates have often been found in hot flash studies [19]. Further studies are needed to directly compare the relative efficacy of escitalopram with hormone therapy in hot flash management regarding long treatment duration; the longest published trial to date is only 12 weeks.

\section{REFERENCES}

[1] Pearce, J., Hawton, K. and Blake, F. (1995) Psychological and sexual symptoms associated with the menopause and the effects of hormone replacement therapy. British Journal of Psychiatry, 167, 163-173. doi:10.1192/bjp.167.2.163

[2] Dennerstein, L. (1996) Well-being, symptoms and the menopausal transition. Maturitas, 23, 147-157. doi:10.1016/0378-5122(95)00970-1

[3] Rossouw, J., Anderson, G., Prentice, R., LaCroix, A., Kooperberg, C., Stefanick, M., Jackson, R., Beresford, S., Howard, B., Johnson, K., Kotchen, J. and Ockene, J. (2002) Risks and benefits of estrogen plus progestin in healthy postmenopausal women: Principal results from the women's health initiative randomized controlled trial. Journal American Medical Association, 288, 321-333. doi:10.1001/jama.288.3.321

[4] Dobkin, D., Menza, M., Allen, L., Marin, H., Bienfait, K., Tiu, J. and Howarth, J. (2009) Escitalopram reduces hot flashes in nondepressed menopausal women: A pilot study. Annals of Clinical Psychiatry, 21, 70-76.

[5] Vickers, M., MacLennan, A., Lawton, B., Ford, D., Martin, J., Meredith, S., DeStavola, B., Rose, S., Dowell, A., Wilkes, H., Darbyshire, J. and Meade, T. (2007) Main morbidities recorded in the women's international study of long duration oestrogen after menopause (WISDOM): A randomised controlled trial of hormone replacement therapy in postmenopausal women. British Medical Journal, 335, 239-244. doi:10.1136/bmj.39266.425069.AD

[6] Rodriguez, C., Patel, A., Calle, E., Jacob, E. and Thun, M. (2001) Estrogen replacement therapy and ovarian cancer mortality in a large prospective study of US women. Journal American Medical Association, 285, 1460-1465. doi:10.1001/jama.285.11.1460

[7] Shumaker, S., Legault, C., Rapp, S., Thal, L., Wallace, R.,
Ockene, J., Hendrix, S., Jones, B.r., Assaf, A., Jackson, R., Kotchen, J., Wassertheil-Smoller, S. and WactawskiWende, J. (2003) Estrogen plus progestin and the incidence of dementia and mild cognitive impairment in postmenopausal women: The women's health initiative memory study: A randomized controlled trial. The Journal of American Medical Association, 289, 2651-2662. doi:10.1001/jama.289.20.2651

[8] Fletcher, S. and Colditz, G. (2002) Failure of estrogen plus progestin therapy for prevention. The Journal of American Medical Association, 288, 366-368. doi:10.1001/jama.288.3.366

[9] Nelson, H., Humphrey, L., Nygren, P., Teutsch, S. and Allan, J. (2002) Postmenopausal hormone replacement therapy: Scientific review. The Journal of American Medical Association, 288, 872-881. doi:10.1001/jama.288.7.872

[10] Boraz, M.A., Simkin-Silverman, L.R., Wing, R.R., Meilahn, E.N. and Kuller, L.H. (2001) Hormone replacement therapy use and menopausal symptoms among women participating in a behavioral lifestyle intervention. Preventive Medicine, 33, 108-114. doi:10.1016/S0091-7435(01)80006-2

[11] Imai, A., Matsunami, K. and Takagi, H., Ichigo, S. (2012) New generation nonhormonal management for hot flashes. Gynecologic Endocrinology, in press. doi: 10.3109/09513590.2012.705380

[12] Loprinzi, C., Sloan, J., Stearns, V., Slack, R., Iyengar, M., Diekmann, B., Kimmick, G., Lovato, J., Gordon, P., Pandya, K., Guttuso, T.J., Barton, D. and Novotny, P. (2009) Newer antidepressants and gabapentin for hot flashes: An individual patient pooled analysis. Journal of Clinical Oncology, 27, 2831-2837. doi:10.1200/JCO.2008.19.6253

[13] Pinkerton, J., Stovall, D. and Kightlinger, R. (2009) Advances in the treatment of menopausal symptoms. Women's Health, 5, 361-384. doi:10.2217/whe.09.31

[14] Sideras, K. and Loprinzi, C. (2010) Nonhormonal management of hot flashes for women on risk reduction therapy. Journal of National Comprehensive Cancer Network, 8, 1171-1179.

[15] Loprinzi, C., Qin, R., Balcueva, E., Flynn, K., Rowland, K.J., Graham, D., Erwin, N., Dakhil, S., Jurgens, D. and Burger, K. (2010) Phase III, randomized, double-blind, placebo-controlled evaluation of pregabalin for alleviating hot flashes, N07C1. Journal of Clinical Oncology, 28, 641-647. doi:10.1200/JCO.2009.24.5647

[16] Freeman, E., Guthrie, K., Caan, B., Sternfeld, B., Cohen, L., Joffe, H., Carpenter, J., Anderson, G., Larson, J., Ensrud, K., Reed, S., Newton, K., Sherman, S., Sammel, M. and LaCroix, A. (2011) Efficacy of escitalopram for hot flashes in healthy menopausal women: A randomized controlled trial. The Journal of American Medical Association, 305, 267-274. doi:10.1001/jama.2010.2016

[17] Soares, C., Arsenio, H., Joffe, H., Bankier, B., Cassano, P., Petrillo, L. and Cohen, L. (2006) Escitalopram versus ethinyl estradiol and norethindrone acetate for symptomatic peri- and postmenopausal women: Impact on depression, vasomotor symptoms, sleep, and quality of life. Menopause, 13, 780-786. 
doi:10.1097/01.gme.0000240633.46300.fa

[18] Soares, C., Thase, M., Clayton, A., Guico-Pabia, C., Focht, K., Jiang, Q., Kornstein, S., Ninan, P., Kane, C. and Cohen, L. (2010) Desvenlafaxine and escitalopram for the treatment of postmenopausal women with major depressive disorder. Menopause, 17, 700-711.

[19] Sloan, J., Loprinzi, C., Novotny, P., Barton, D., Lavasseur, B. and Windschitl, H. (2001) Methodologic lessons learned from hot flash studies. Journal of Clinical Oncology, 19, 4280-4290. 